\title{
Sensing Magnons with a Superconducting Qubit
}

\author{
A new method can detect a steady-state population of magnons in a \\ crystal.
}

By Erika K. Carlson

E arlier this year, a team of researchers at the University of Tokyo showed that they could use a superconducting qubit to detect the presence of a single magnon-a magnetic excitation associated with electron spins. Now, this group, led by Sam Wolski, has demonstrated that the same technology can detect a steady-state population of magnons with a greater sensitivity than past methods [1]. The development is an example of how quantum technologies, such as qubits, can lead to improvements in sensing and other applications.

For their experiments, Wolski and colleagues placed a spherical crystal of yttrium-iron-garnet and a superconducting qubit inside a copper cavity. They created an oscillating microwave magnetic field in the cavity that excited a number of magnons in the crystal. The researchers coupled both the qubit and the crystal to a resonant mode of the cavity, which also effectively coupled the qubit and crystal to each other.

The coupling makes the qubit's quantum state dependent on

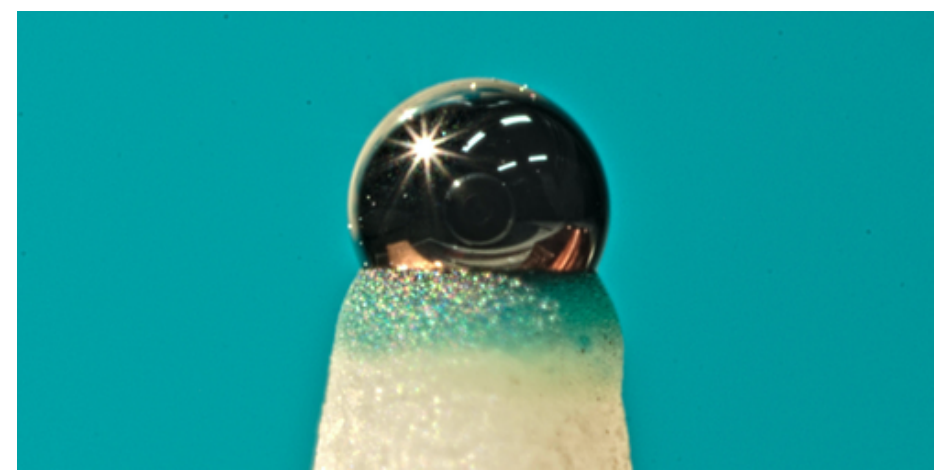

Credit: S. P. Wolski et al. [1] the number of magnons in the crystal, so measuring this state allowed the researchers to infer how many magnons were in the crystal. They showed that they could detect magnon populations that are many orders of magnitude smaller than those detectable with other methods. This increased sensitivity, the researchers say, may be useful in developing transducers for quantum networks and for quantum information processing systems. It may even assist in searches for axions, a candidate dark matter particle, by potentially allowing researchers to spot tiny interactions these hypothetical particles may have with electron spins in a system.

Erika K. Carlson is a Corresponding Editor for Physics based in New York City.

\section{REFERENCES}

1. S. P. Wolski, "Dissipation-based quantum sensing of magnons with a superconducting qubit," Phys. Rev. Lett. 125, 117701 (2020). 